\title{
Database application model and its service for drug discovery in Model-driven architecture
}

\author{
Noriko Etani
}

\section{Correspondence:}

etani.noriko.2s@kyoto-u.ac.jp Graduate School of Medicine, Kyoto University/CREST, Japan Science and Technology Agency. 36-1 Yoshida-honmachi, Sakyo-ku, 6068501 Kyoto, Japan

\section{照 Springer}

\begin{abstract}
Big data application has many data resources and data. In the first stage of software engineering, a service overview or a system overview cannot be seen. In this paper, we propose that two processes of "Big data analytics" and "Implementation of data modeling" should be collaborated with Model-driven architecture (MDA). Data modeling with those two process in MDA should be repeated fast in order to verify the data model and to find a new data resource for a service. Our first research goal of big data application is to predict side effect of drug which is one of screening methods in drug discovery. This prediction model is constructed with data mining methods at the intersection of statistics, machine learning and database system. Moreover, a new service for drug discovery by new uses for old drugs can be found in data modeling and developed. We demonstrate that the prediction model and the data model for drug discovery are implemented as a prototype system to verify those models and their practicality.
\end{abstract}

Keywords: PLS regression analysis, Discriminant analysis, Support vector machine, Prediction model, Drug side effect, Drug discovery, Model-driven architecture (MDA)

\section{Introduction}

The key properties of big data are characterized as volume, velocity, variety, veracity, and value [1]. The value (business value) is crucial to offer services for business. To enhance the value, the veracity, which is about data understandability, is useful for people's decision making. Here, the "veracity" of big data properties is an important issue to develop an application for drug discovery because all of the clinical data is not offered and the data in drug discovery is sparse. Moreover, in the first stage of software engineering, a service overview or a system overview cannot be seen because big data application has many data resources and data. Therefore, we propose that two processes of "Big data analytics" and "Implementation of data modeling" should be collaborated with Model-driven architecture (MDA) [2] in order to repeat data modeling fast and find a new service shown in Fig. 1. MDA by the Object Management Group (OMG) is a software design approach for the development of software systems. This architecture has three layers for abstraction of data modeling because software solutions can be automatically derived from models by "technology independence". "Domain model" is modeling the actual existence of a domain. It is called "Computation Independent Model (CIM)". "Logical system model" is modeling a service with domain model, in which the components of a system interact with

C 2015 Etani. Open Access This article is distributed under the terms of the Creative Commons Attribution 4.0 International License (http://creativecommons.org/licenses/by/4.0), which permits unrestricted use, distribution, and reproduction in any medium, provided you give appropriate credit to the original author(s) and the source, provide a link to the Creative Commons license, and indicate if changes were made. The Creative Commons Public Domain Dedication waiver (http://creativecommons. org/publicdomain/zero/1.0/) applies to the data made available in this article, unless otherwise stated. 


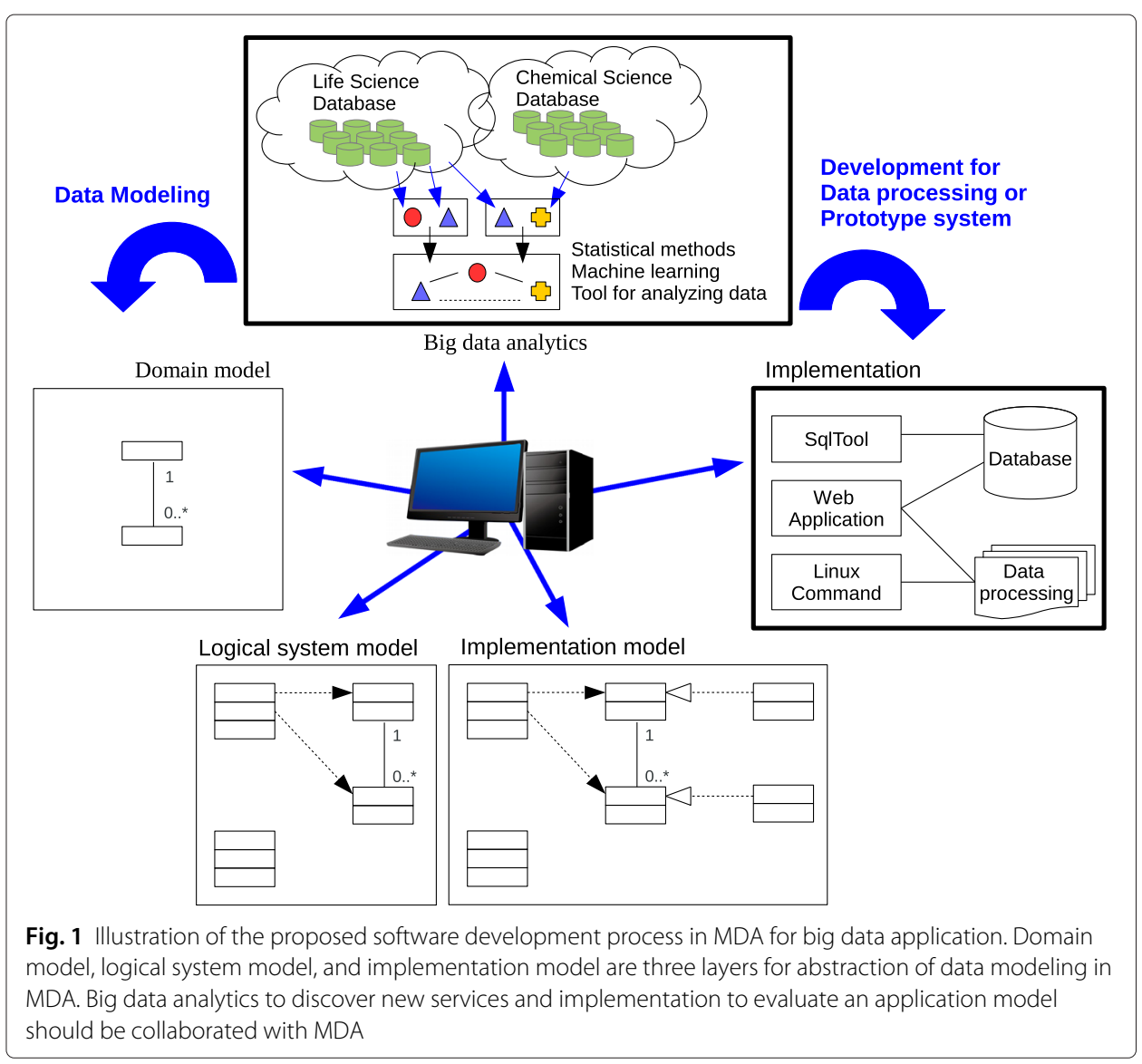

each other. It is called "Platform Independent Model (PIM)" "Implementation model" is modeling a specified system which logical system model can work with a specified technology and platform, which examples are Linux, Web application and SQL database. It is called "Platform Specific Model (PSM)". Those 5 processes are encapsulated and operated in parallel.

In this paper, we develop database application model and its service for side effect prediction of drug, which is our first research goal of big data application, and for drug discovery, which finds new uses for old drugs, introducing the proposed software development process in MDA into our research process. Drug side effect prediction is one of screening methods in drug discovery. The drug candidates can be ranked by this prediction.

The following sections of the paper are organized as follows: the "Background and literature review" section will describe background of drug discovery and related works of side effect prediction and gene-drug discovery. The "Research design and methodology" section will outline the approach taken by the proposed research of side effect prediction and drug discovery. The performance of the proposed system will be demonstrated in the "Results and discussion" section. Finally, the "Conclusions" section will describe a direction for future.

\section{Background and literature review}

In recent years, a research on drug discovery to find the new medical indication from the old drugs and to redevelop them as a treatment for another disease has attracted attention 
as drug re-positioning in order to minimize cost and a development period for efficiency [3]. One reason is complex biological processes of most human diseases which the 'onedrug-one-gene' approach is not effective to treat [4]. In addition, the official report on the side effects of prescription drugs that have increased dramatically over the past decade. In 2011, U.S. Food and Drug Administration (FDA) has received about 500,000 reports of health hazards and the death related to medical products per year [5]. In considering the safety of the drug, the prediction of side effect in drug discovery is an important issue for drug screening. Then, the personalized medicine is expected to develop new drugs and treatments, and to avoid side effect using Single Nucleotide Polymorphism (SNP) which will contribute to identifying genes related to the diseases [6]. More than one SNP are in a gene. Here, previous works on potential side effect prediction of drug candidates and drug discovery with SNPs using Big Data are reviewed.

The research work to predict the side effects of drugs is done by the relationship between chemical and target protein. Yamanishi et al. [7] proposed a kernel regression model as computational model. This method predicted a potential side effect profiles based on the chemical structures and the information on the target protein. This method ranked first one of the side effects of $41.7 \%$ (275 drugs) in 658 drugs, and ranked a correct side effect among the top five scoring for $70.0 \%$ (461 drugs) in 658 drugs. Kuhn et al. [8] focused on similarities in the side effects of drugs. The clinical data on chemical and protein were collected. And by combining drug-target protein and drug-side effect relations, overrepresented target protein-side effect pars are identified. 732 of 1428 side effects were predicted to be mainly caused by individual proteins. 137 of 732 side effects were proved by pharmacological or phenotypic existing data. From the results of these studies, classification and regression characteristics of the side effect are found. However, these studies will fall into a lack of an overall service-based solution because all data for the side effect prediction are not always prepared. Although the above-mentioned 2 studies focused on potential side effect, we focus not only on side effect, but also on its incidence. Therefore, we propose the comprehensive prediction model of drug side effect and its incidence using the data mining approach at the intersection of statics, machine learning and database system considering classification and regression characteristics of the side effect. And the gene information of target protein and the chemical (drug) - protein interaction are considered in this paper.

As for gene-drug discovery, Riken developed a method of gene-drug discovery for rheumatoid arthritis [9]. Genome-wide association study discovered 101 disease genes of rheumatoid arthritis from SNPs. A new method proved that 101 disease genes were connected with target proteins of the existing rheumatoid arthritis drugs through proteinprotein interaction network [10]. Moreover, it indicated that the existing drugs for other diseases were connected to disease genes of rheumatoid arthritis, and that those existing drugs were possible drug candidates for new uses of rheumatoid arthritis. So, we considered this results in the clue of drug discovery, and propose database application model to discover the new drug candidates by new uses of the old drugs.

\section{Research design and methodology}

Data resources

Data from the different databases are publicly available, and collected useful for data analytics as follows: 
- KEGG: a database resource of biological system [11]. Human diseases, disease genes, drugs and ATC code [12] are extracted.

- JSNP: a repository of Japanese SNP data [13-15]. Diseases, associated SNPs, and disease genes are extracted.

- STITCH 4.0: a database of protein-chemical interactions [16]. Confidence score of chemical (drug) - protein interaction is extracted.

- SIDER 2: a side effect resource to capture phenotypic effects of drugs [17, 18]. Chemical (drug), ATC code, drug side effect, and its incidence are extracted.

- PubChem: a database of chemical molecules [19]. Chemical ID according to drug number in the KEGG database is extracted.

\section{Data features}

Drug

In drug discovery, drug candidates are selected by the interaction with target proteins including disease genes of human organ or system. So, the properties of drug are characterized as biological, anatomical, genetic, and clinical features. Pinpoint data in each feature is selected. Confidence score of chemical (drug) - protein interaction as biological feature, the first level of ATC code as anatomical feature, Gene ID of disease gene as genetic feature, and side effect and its incidence as clinical feature are utilized. As for ATC code, the Anatomical Therapeutic Chemical (ATC) Classification System uses it, which is controlled by the World Health Organization Collaborating Center for Drug Statistics Methodology, to classify drugs into five different groups according to the organ or system on which they act and their therapeutic and chemical characteristics [12, 20]. Here, the first level, that is the anatomical main group according to the organ or system which drugs act, is utilized. It consists of one letter. There are 14 anatomical groups as follows: (A) Alimentary tract and metabolism, (B) Blood and blood forming organs, (C) Cardiovascular system, (D) Dermatologicals, (G) Genito urinary system and sex hormones, (H) Systemic hormonal preparations, excluding sex hormones and insulins, (J) Antiinfectives for systemic use, (L) Antineoplastic and immunomodulating agents, (M) Musculo-skeletal system, (N) Nervous system, (P) Antiparasitic products, insecticides and repellents, (R) Respiratory system, (S) Sensory organs, and (V) Various.

There are 334 drugs which show their incidences of more than $0.1 \%$ in the SIDER 2 database. The number of the side effect which is extracted from the SIDER 2 database is 2326. The highest scores for these 334 drugs as biological feature are extracted from the STITCH 4.0 database. 76 drugs among 334 drugs have the disease genes in the KEGG database. The number of the gene which is extracted from the KEGG database is 335 .

\section{Gene}

2385 disease genes, in which 801 genes have the drugs, are extracted from the KEGG database. 11490 SNPs and 6513 genes, whose diseases are also nominated in the KEGG database, are extracted from the JSNP database.

\section{Disease}

717 diseases, in which 174 diseases have the drugs, are extracted from the KEGG database. 64 diseases, which are also nominated in the KEGG database, are found in JSNP database. 


\section{Prediction model \\ Side effect prediction}

The first level of ATC code in 334 drugs and Gene ID of the disease gene in 76 drugs can classify the side effects. As for ATC code, Table 1 shows the number of the side effect which is classified by the first level of ATC code. For example, "(L) Anti-neoplastic and immunomodulating agents" of ATC code can find 970 side effects which are including the typical symptoms of leukemia, lymphopenia and thrombocytopenia. As for the disease gene, side effects can be derived from the drug which is interacted with the disease gene. For example, the "HLA-DQA1" gene, which provides instructions for making a protein that plays a critical role in the immune system, can find 130 side effects extracted from the drugs of propanolol, simvastatine, dipyridamole and azathioprine which have it as the disease gene.

\section{Incidence prediction}

As for clustering method, discriminant analysis using regression formula is utilized to classify the data with 2 classes. In Table 2, "SCORE", "ATC", and "Gene ID" in pinpoint data are the explanatory variables. The response variable is "Incidence". All of those explanatory variables are not always prepared because the data is sparse. So, 7 dataset patterns of pinpoint data are prepared to be able to predict side effect incidence even if some of those explanatory variables are missing. A mark on Table 2 indicates the existing pinpoint data.

Firstly, each dataset is analyzed with regression analysis of a statistical method. Secondly, discriminant analysis (DA) sets a discriminant criterion from the results by the regression analysis, and classifies the group by the criterion. Linear discriminant formula calculates a value which can classify the group. Then, each DA value is divided into the positive group or the negative group. In the regression analysis, single regression analysis in case of one explanatory variable is available, and partial least squares (PLS) regression analysis in case of more than one explanatory variable is available because a problem of multicollinearity may be occurred and it is difficult to decide the group border when one explanatory variable has correlation with another explanatory variable. When DA using multiple regression analysis is executed in a case of Dataset No. 4 shown in Table 2, Fig. 2 shows that it is difficult to decide the group boundary because there is variability around the threshold which value is " 0 ". Instead of multiple regression analysis, PLS regression analysis is introduced. As a result, Fig. 3 shows that it is easy to decide the group boundary at $34.7 \%$.

Table 1 The number of side effect classified by the first level of ATC code

\begin{tabular}{llll}
\hline ATC & The number of side effect & ATC & The number of side effect \\
\hline ALL & 2326 & L & 970 \\
A & 642 & M & 652 \\
B & 264 & N & 1704 \\
C & 632 & P & 113 \\
D & 379 & R & 380 \\
G & 523 & S & 914 \\
H & 132 & V & 191 \\
J & 702 & &
\end{tabular}

"ATC" indicates the first level of ATC code. "The number of side effect" shows the results of side effect classification by the first level of ATC code 
Table 2 Dataset of pinpoint data in Drug properties

\begin{tabular}{llllll}
\hline Dataset No. & SCORE & ATC & Gene ID & Incidence & Items \\
\hline 1 & $\circ$ & $\circ$ & & $\circ$ & 358 \\
2 & $\circ$ & & & $\circ$ & 262 \\
3 & & $\circ$ & $\circ$ & $\circ$ & 214 \\
4 & $\circ$ & $\circ$ & $\circ$ & $\circ$ & 469 \\
5 & $\circ$ & & $\circ$ & $\circ$ & 334 \\
6 & & $\circ$ & $\circ$ & 469 \\
7 & & & $\circ$ & $\circ$ & 334 \\
\hline
\end{tabular}

"Dataset No." indicates 7 dataset patterns with pinpoint data of each drug property. "SCORE" indicates confidence score of chemical (drug) - protein (disease gene) interaction as biological feature. "ATC" indicates the first level of ATC code as anatomical feature. "Gene ID" indicates Gene ID of disease gene as gene feature. "Incidence" indicates side effect incidence as clinical feature. "Items" indicates the number of the data in each dataset

This model is described as follows: An example formula of PLS regression model in Dataset No. 4 shown in Table 2 can be represented as

$$
y^{\prime}=a_{1}{ }^{*} \mathrm{SCORE}+a_{2}{ }^{*} \mathrm{ACT}+a_{3}{ }^{*} \text { Gene ID }+b
$$

where $y^{\prime}$ is predicted value, $a_{1}$ is coefficient of explanation variable SCORE, $a_{2}$ is coefficient of explanation variable ATC, $a_{3}$ is coefficient of explanation variable Gene ID and $b$ is intercept. A formula of PLS-DA can be represented as

$$
\mathrm{f}(\mathrm{x})=y-y^{\prime} .
$$

$y$ is observed value. Discrimination rule is as follows: If $f(x) \geq 0$ Then $\operatorname{sgn}[f(x)]=1$ and If $f(x)<0$ Then $\operatorname{sgn}[f(x)]=-1$. Finally, each data is analyzed with DA and classified with 2 classes until one group in one class cannot be classified. As a result, Table 3 shows the clustering. And it indicates that the clustering is available with almost $100 \%$ correct rate.

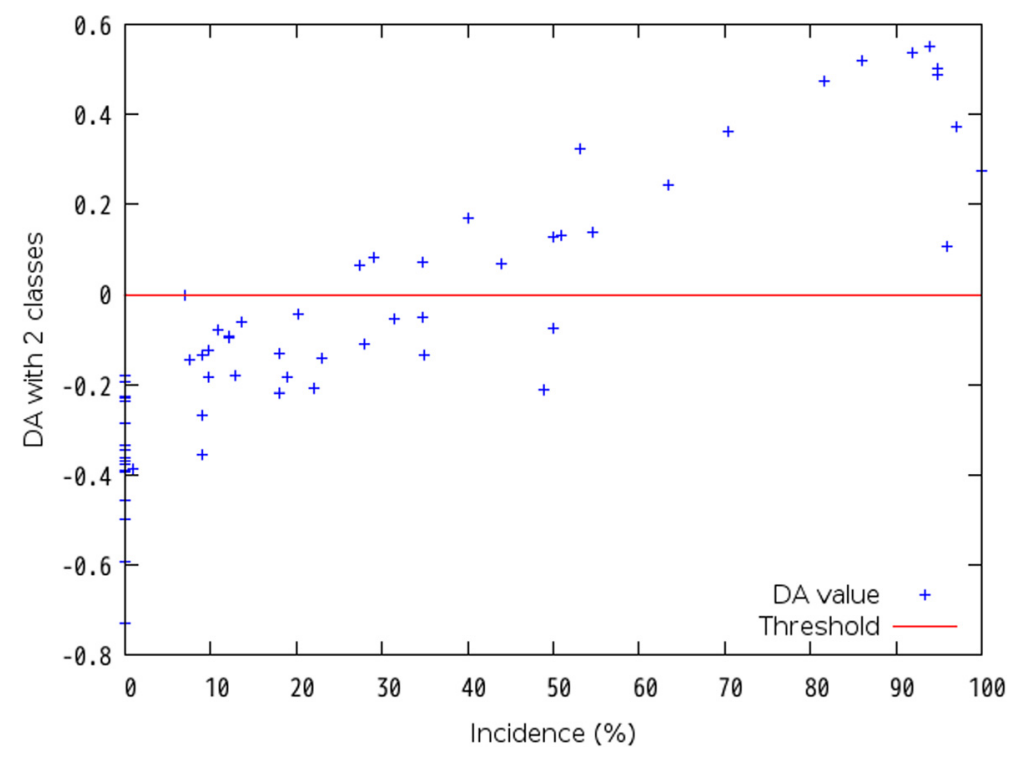

Fig. 2 Discriminant Analysis with 2 classes by Multiple Regression formula in Dataset No. 4. This shows a result of the first grouping of the data in Dataset No. 4 shown in Table 2 by discriminant analysis with 2 classed using multiple regression formula. The threshold value is "0" to classify DA values with a positive class or a negative class 


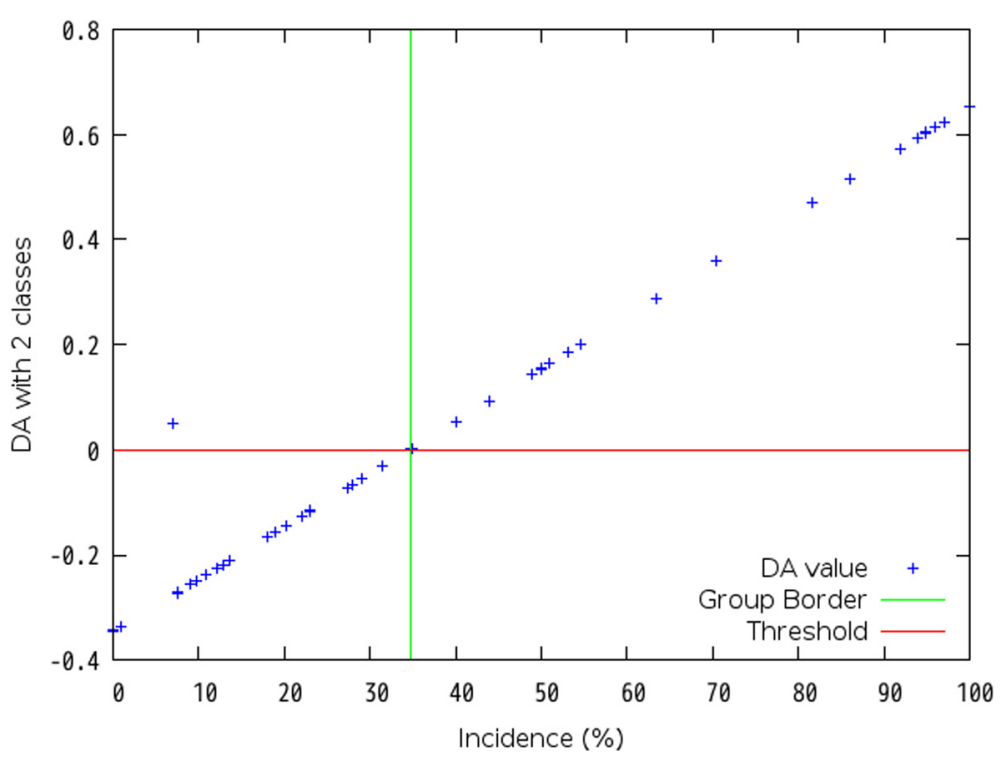

Fig. 3 Discriminant Analysis with 2 classes by PLS regression formula in Dataset No. 4. This shows a result of the first grouping of the data in Dataset No. 4 shown in Table 2 by discriminant analysis with 2 classed using PLS regression formula. The threshold value is " 0 " to classify DA values with a positive class or a negative class. $34.7 \%$ can be a group boundary for clustering

As for prediction method, Support Vector Machine (SVM) is supervised learning models with associated learning algorithms that analyze data and recognize patterns. A training algorithm of SVM learns the rules and creates the learning model. SVM takes a set of input data and predicts with the learning model. LibSVM Version 3.18 [21] for a machine learning library is available. The example model in Dataset No. 4 shown in Table 2 is described as follows: When there are $n$ samples of Dataset No. 4, input space can be represented as

$$
X=\left(\operatorname{SCORE}_{1}, A T C_{1}, \text { GeneID }_{1}\right), \cdots,\left(S C O R E_{n}, A T C_{n}, \text { GeneID }_{n}\right) .
$$

Table 3 Evaluation of clustering

\begin{tabular}{lrllrl}
\hline Dataset No. & Clustering & Correct rate (\%) & Dataset No. & Clustering & Correct rate (\%) \\
\hline 1 & $41-100 \%$ & 99 & 5 & $34.7-100 \%$ & 100 \\
2 & $55-100 \%$ & 96 & & $27.4-100 \%$ & 100 \\
& $29-100 \%$ & 100 & 6 & $12.1-100 \%$ & 100 \\
3 & $9-100 \%$ & 100 & $53.2-100 \%$ & 95 \\
& $70.3-100 \%$ & 100 & $51-100 \%$ & 100 \\
& $36-100 \%$ & 99 & $50-100 \%$ & 100 \\
4 & $18-100 \%$ & 100 & $34.7-100 \%$ & 100 \\
& $81.7-100 \%$ & 100 & $27.4-100 \%$ & 100 \\
& $63.5-100 \%$ & 100 & $18-100 \%$ & 100 \\
& $34.7-100 \%$ & 100 & $12.1-100 \%$ & 100 \\
& $27.4-100 \%$ & 100 & $81.7-100 \%$ & 100 \\
& $12.1-100 \%$ & 100 & $49-100 \%$ & 100 \\
& $94-100 \%$ & 100 & & $18-100 \%$ & 100 \\
\hline
\end{tabular}

"Dataset No". indicates 7 dataset patterns of pinpoint data shown in Table 2. "Clustering" indicates the group divided by DA

"Correct rate" indicates to what extent each example can be classified with each correct clustering 
Output domain can be represented as $Y=\{1,-1\}$. A formula of class classification can be represented as $f(x)=<w \cdot x>+b$ where $w$ is weight vector, and $b$ is bias. Classification rule is as follows: If $f(x) \geq 0$ Then $\operatorname{sgn}[f(x)]=1$ (positive class) and If $f(x)<0$ Then $\operatorname{sgn}[f(x)]=-1$ (negative class). Table 4 shows evaluation of each learning model for prediction by SVM. It indicates that the prediction models are available with almost $100 \%$ correct rate.

As for prediction procedure, firstly, new dataset is classified into one dataset pattern shown in Table 2. Secondly, in descending order of the clustering, SVM will response true or false to predict the lower value of the prediction interval. In a case of Dataset No. 2 shown in Table 2, there are 3 intervals shown in Table 3. When a parameter of SVM is the lower value of "55" and SVM responses false, the parameter is changed to the next interval's lower value of "29". When SVM responses true in this parameter, it is decided that side effect incidence will be from $29 \%$ to $100 \%$ in the approximated interval. When SVM responses false with the last parameter of "9", it is decided that side effect incidence will be from $0.1 \%$ to $100 \%$ in the approximated interval.

\section{Data modeling}

Data modeling for side effect prediction is done, and its model is implemented to verify its service. After that, the data concerning gene is collected from the KEGG database and the JSNP database. In addition to side effect prediction, new services for drug discovery are found in data modeling. Here, the result of modeling database application in each model is described.

\section{Domain model}

Figure 4 shows a static view of the objects in an application for side effect prediction and drug discovery. There are 6 domain objects and their relations. 6 domain objects consists

Table 4 Evaluation of learning model

\begin{tabular}{|c|c|c|c|c|c|}
\hline Dataset No. & Clustering & Correct rate (\%) & Dataset No. & Clustering & Correct rate (\%) \\
\hline \multirow[t]{2}{*}{1} & $41-100 \%$ & 100 & 5 & $34.7-100 \%$ & 100 \\
\hline & $0.1-100 \%$ & 100 & & $27.4-100 \%$ & 99.5 \\
\hline \multirow[t]{4}{*}{2} & $55-100 \%$ & 23 & & $12.1-100 \%$ & 99.6 \\
\hline & $29-100 \%$ & 95 & & $0.1-100 \%$ & 100 \\
\hline & $9-100 \%$ & 40 & 6 & $53.2-100 \%$ & 100 \\
\hline & $0.1-100 \%$ & 100 & & $51-100 \%$ & 100 \\
\hline \multirow[t]{4}{*}{3} & $70.3-100 \%$ & 100 & & $50-100 \%$ & 100 \\
\hline & $36-100 \%$ & 90 & & $34.7-100 \%$ & 100 \\
\hline & $18-100 \%$ & 20 & & $27.4-100 \%$ & 98 \\
\hline & $0.1-100 \%$ & 86 & & $18-100 \%$ & 98 \\
\hline \multirow[t]{6}{*}{4} & $81.7-100 \%$ & 100 & & $12.1-100 \%$ & 99 \\
\hline & $63.5-100 \%$ & 100 & & $0.1-100 \%$ & 100 \\
\hline & $34.7-100 \%$ & 100 & 7 & $81.7-100 \%$ & 100 \\
\hline & $27.4-100 \%$ & 100 & & $49-100 \%$ & 100 \\
\hline & $12.1-100 \%$ & 100 & & $18-100 \%$ & 99 \\
\hline & $0.1-100 \%$ & 100 & & $0.1-100 \%$ & 100 \\
\hline 5 & $94-100 \%$ & 100 & & & \\
\hline
\end{tabular}

"Dataset No". indicates 7 dataset patterns of pinpoint data shown in Table 2. "Clustering" indicates the approximated interval of prediction in side effect incidence of drug. "Correct rate" indicates to what extent each example of learning model can meet each correct interval in the prediction 


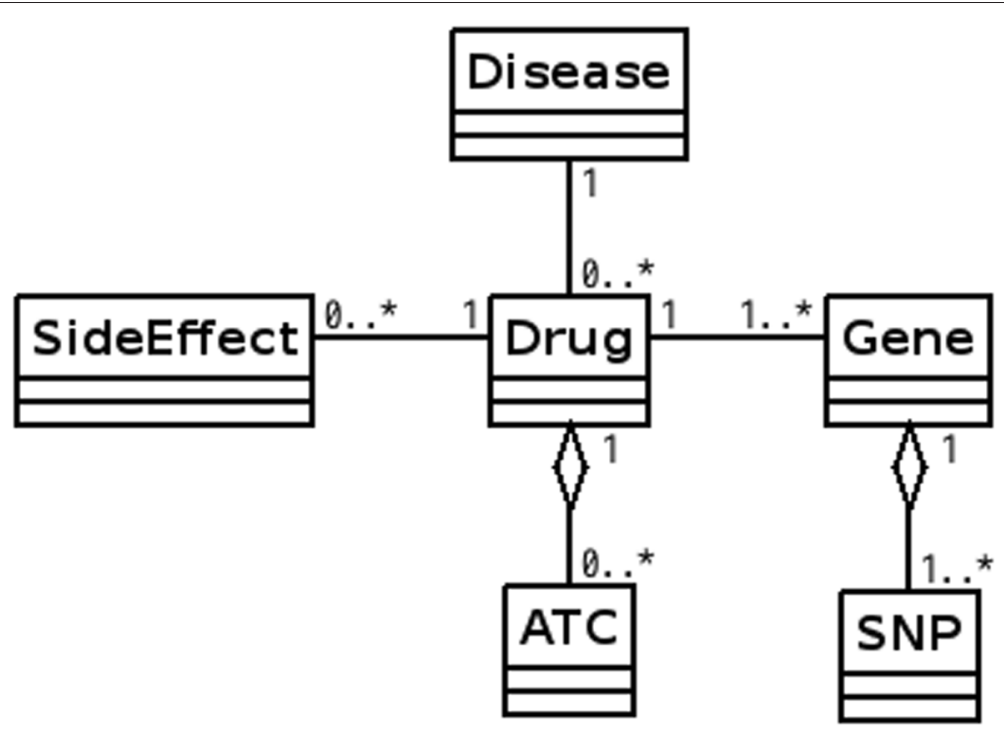

Fig. 4 Domain model. There are 6 domain objects. There are 3 association links among a Drug object, a Gene object, a Disease object and a SideEffect object. There are 2 aggregation links between a Drug object and an ATC object, and between a Gene object and a SNP object. The notation 0..* in the diagram means "zero to many". And the notation $1 . .{ }^{*}$ in the diagram means "one to many"

of a Drug object, an $A T C$ object, a Gene object, a SNP object, a Disease object and a SideEffect object.

Association indicates any logical connection or relationship between objects. There are 3 association links among a Drug object, a Gene object, a Disease object and a SideEffect object. One disease contains zero to many drugs. One drug contains zero to many side effects. And one drug contains one to many genes.

Aggregation indicates the formation of a particular object as a result of one object built as a collection. In aggregation, the contained objects are not strongly dependent on the life cycle of the container. There are 2 aggregation links between a Drug object and an ATC object, and between a Gene object and a SNP object. A Drug object is made up of none or more ATC codes. And a Gene object is made up of one or more SNPs.

\section{Logical system model}

Figure 5 shows a static view of the objects and classes that make up the design and analysis space. There are 7 domain objects. A DrugCandidate object is added to 6 domain objects shown in Fig. 4. They are persistent objects and saved in a database. A Incidence object is temporary object which is not saved anywhere.

For the class model, 4 new classes for services are modeled. An IncidenceCatalog class contains one incidence, and uses "Learning Model Files" from Dataset No. 1 to Dataset No. 7 shown in Table 2. A SideEffectCatalog class contains one to many side effects. Those 2 classes are for drug side effect. A DiseaseClassificationCatalog class contains one to many diseases. A DrugCandidateCatalog class contains one to many drug candidates, and uses "Drug Candidate Files" which consists of Gene ID, Chemical ID, and Score. Those 2 classes are for drug discovery by new uses for old drugs. 


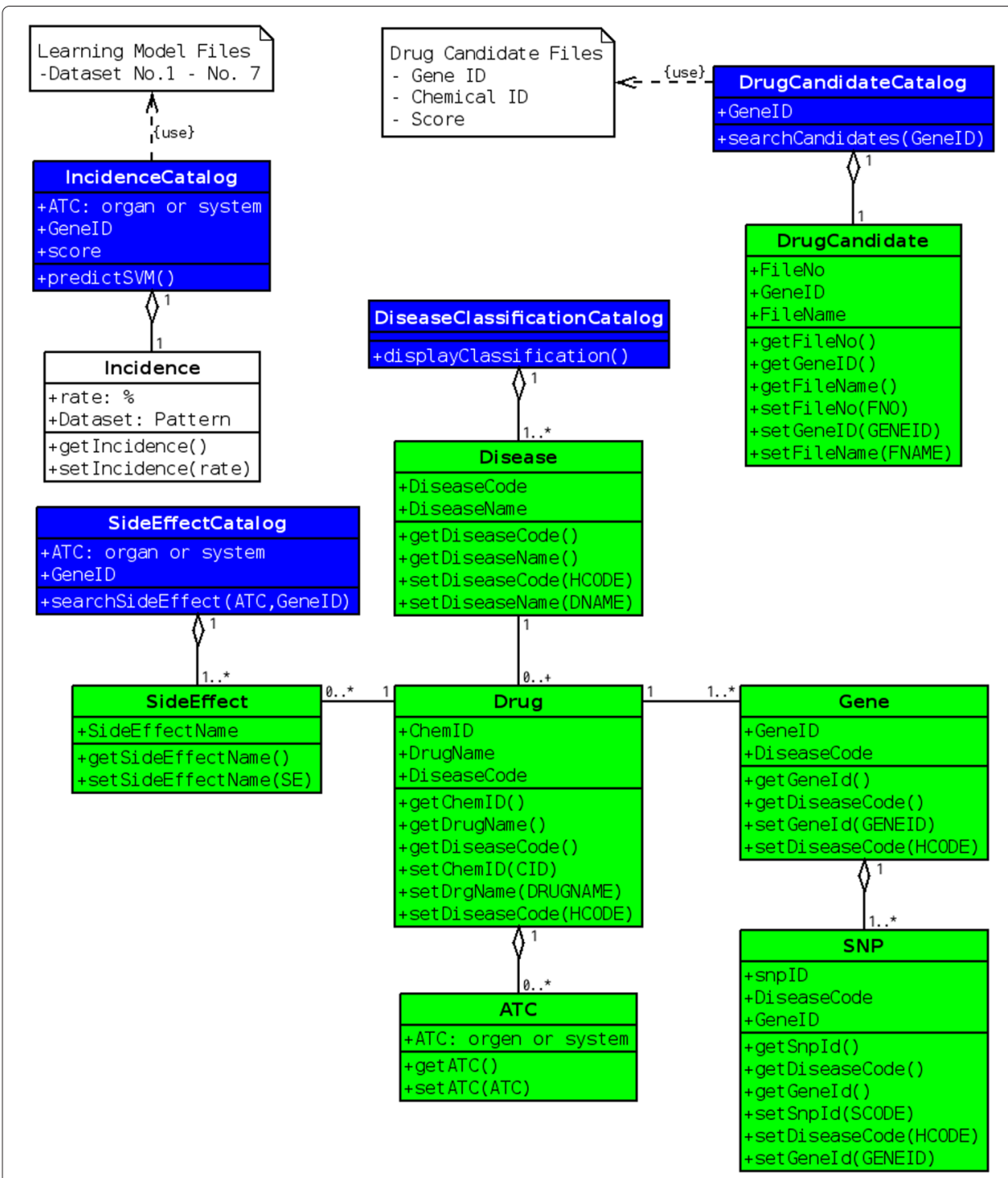

Fig. 5 Logical system model. There are 7 domain objects as persistent objects, which diagrams are green, and one object as temporal object, which diagram is white. For the class model, 4 new classes for services, which diagrams are blue, are modeled. And each attributes and each behavior are described in each object and class

In class design, the class model expresses both the persistent state and the behavior of the system. The state (attributes) are encapsulated in a class, and manipulated to offer services by a class (behaviors). Each attributes and each behaviors are modeled in each class and object.

\section{Implementation model}

Figure 6 describes an implementation-specific solution to satisfy what is specified by the logical system model. Figure 6 shows database application model with web application. There are 6 tables. They realize 6 objects by object-relational mapping. A Disease object is realized by "Disease" table. A Drug object is realized by "Disease" table and "Drug" table. A Gene object is realized by "Disease" table and "Gene" table. A SNP object is realized by 


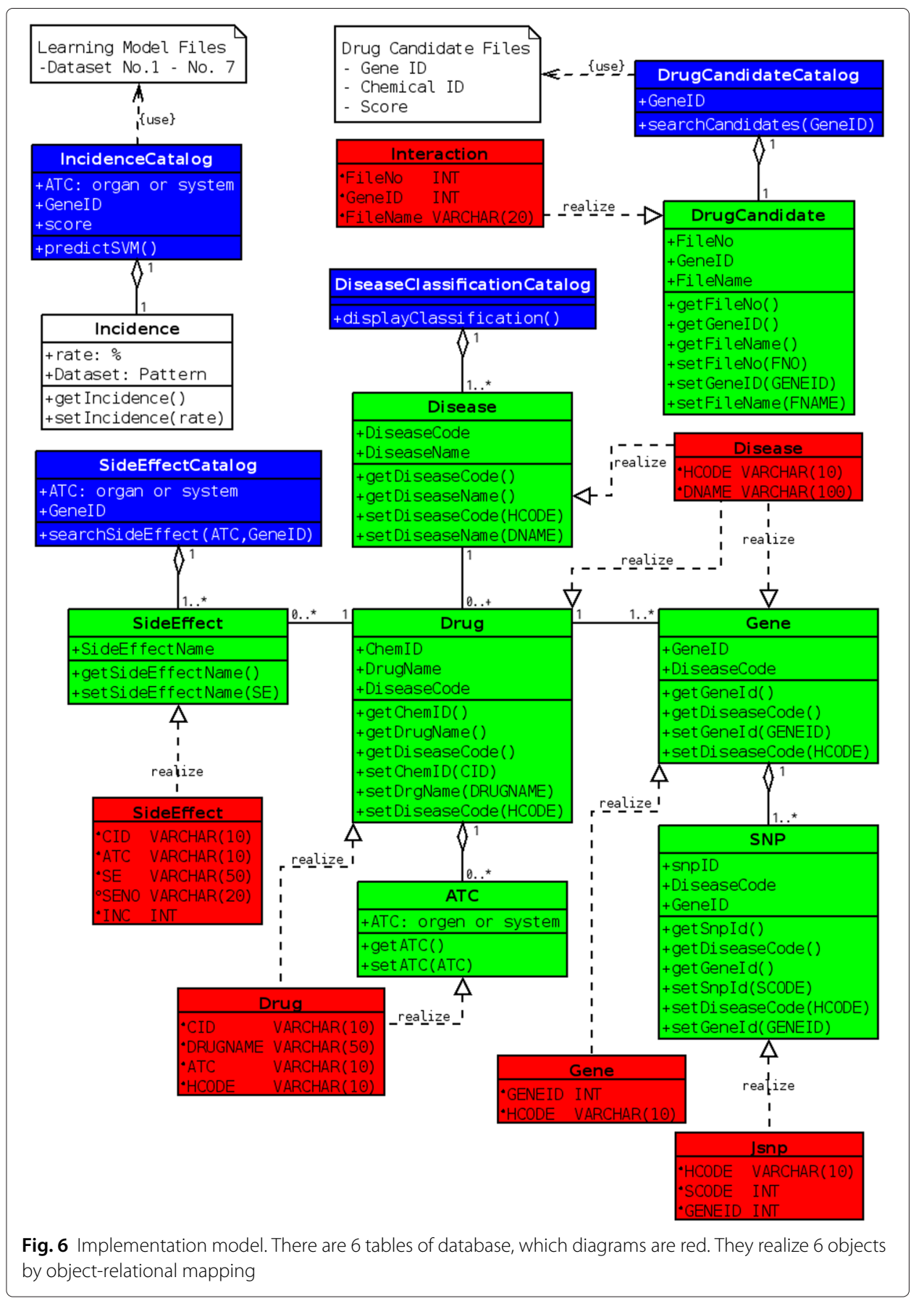

"Jsnp" table. A SideEffect object is realized by "SideEffect" table. A DrugCandidate object is realized by "Interaction" table.

\section{Implementation}

\section{System overview}

Figure 7 shows system overview to realize the implementation model shown in Fig. 6. This system consists of a database with 6 tables and two file groups. A database is accessed with SqlTool and Web application. Both file groups are accessed with Web application. 


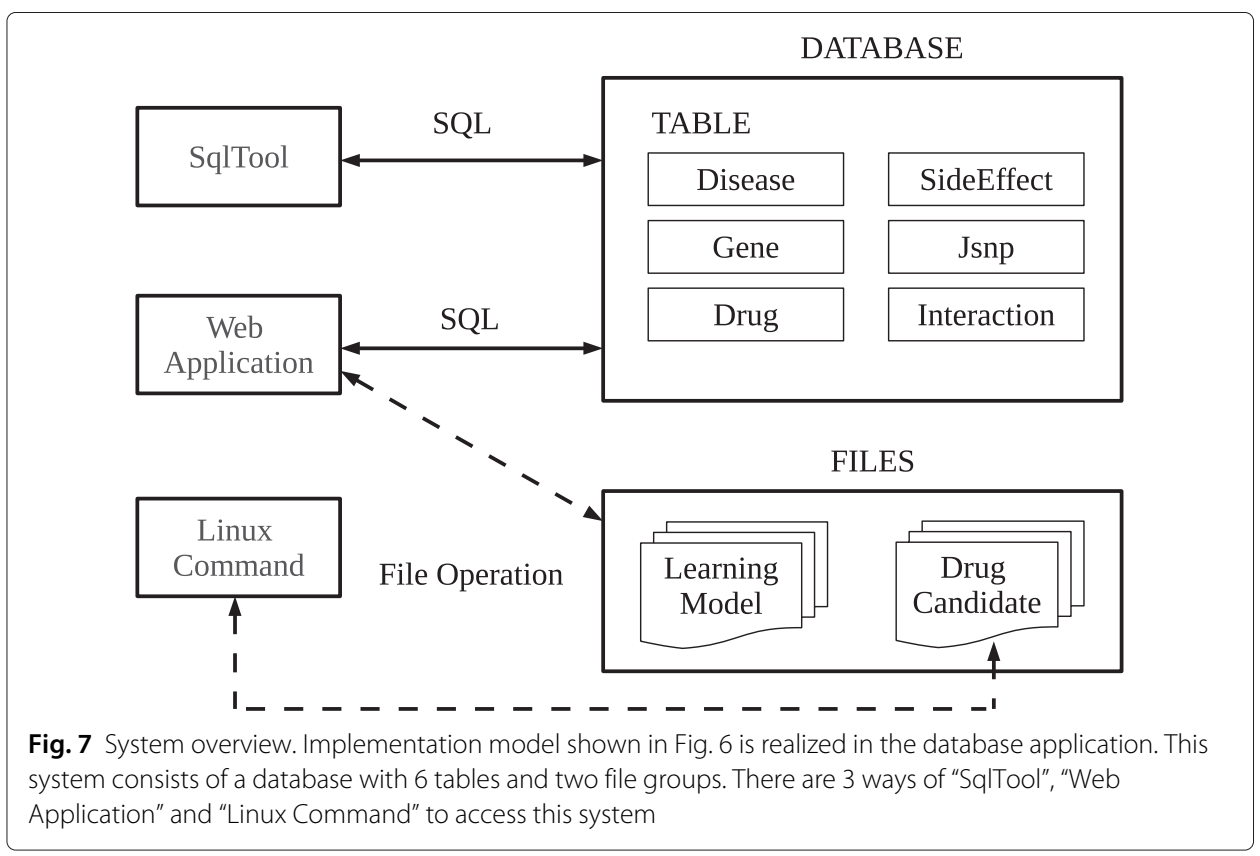

One file group of "Drug Candidate" is also accessed with Linux command. It is freely accessed to search for new interaction pairs by Gene ID and chemicalID. Using this database, the clustering is created by statistical regression analysis and discriminant analysis, and SVM creates the learning model with the clustering. When the data is input in incidence prediction, its incidence is calculated with those learning model files using SVM.

\section{Software overview}

Table 5 shows software development environment. As for Web application, open source is adopted. In order to develop a prototype system to evaluate the models, the domain object of Book Management System (bcat) [22], which is free-ware, is utilized. As a prototype system, Tomcat server can be connected to web browser by port 8080 in the development environment of Java2SE.

Figure 8 shows software overview for Web application. Web server and Servlet/Jsp container are working by Tomcat. Struts framework can work on Servlet/Jsp container.

Table 5 Software development environment. Open source and free ware are publicly available

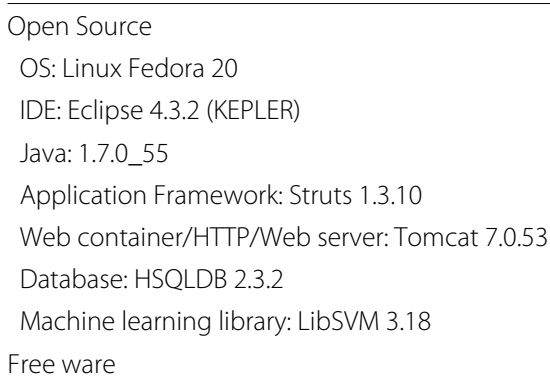




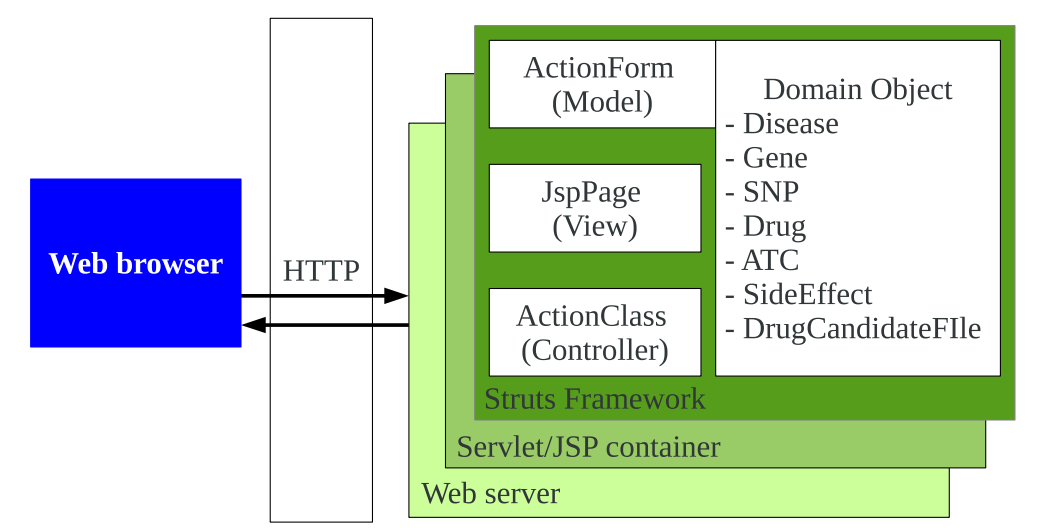

Fig. 8 Software overview for Web Application. The Hypertext Transfer Protocol (HTTP) is an application-level protocol to connect web browser with web application on Tomcat server. ActionForm for model, JspPage for view, and ActionClass for controller to operate 7 domain objects are consisted in software for web application

The prototype System with Struts framework are integrated by three components of ActionForm, ActionClass, and JspPage to operate 7 domain objects.

\section{Application}

Side effect Prediction There are two services of side effect prediction and incidence prediction for Web application. Figure 9 shows operation screens for side effect prediction. When the input data is received, side effects are classified by the input data and shows its result. Figure 10 shows operation screens for incidence prediction. When one dataset of 3 input items is received from input form, its incidence is calculated by LibSVM using class Runtime. Those 3 input items correspond to the explanatory variables of "SCORE", "ATC", and "Gene ID" shown in Table 2, and Equation 3. After that, the result of calculation is displayed.

Drug Discovery by new uses for old drugs There are two approaches. The first approach is to discover drug candidates in new chemicals (drugs)-proteins (disease genes) interactions. A DrugCandidateCatalog object shown in Fig. 6 offers a service to search new chemicals (drugs)-proteins (disease genes) interactions by disease gene. Moreover, when a file group of "Drug Candidate" are directly operated by Linux commands, interactive other genes can be searched by the drugs which are combined with disease genes. The second approach is to discover drug candidates in the disease group related by disease genes and SNPs. Using SqlTool, diseases can be grouped with the common SNP and gene. Diseases which has no drugs can find the drug candidates when other diseases in the same group have the drugs.

\section{Results and discussion}

Side effect prediction

The accuracy in the proposed Web application is evaluated using 9 drugs, which are not nominated in the SIDER 2 database and extracted from the side effect list of "The Internet Drug Index" [23].

As for side effect prediction, $93 \%$ (197 side effects) in 211 side effects can be predicted when ATC code is classified. The reasons why $7 \%$ (14 side effects) in 211 side effects 


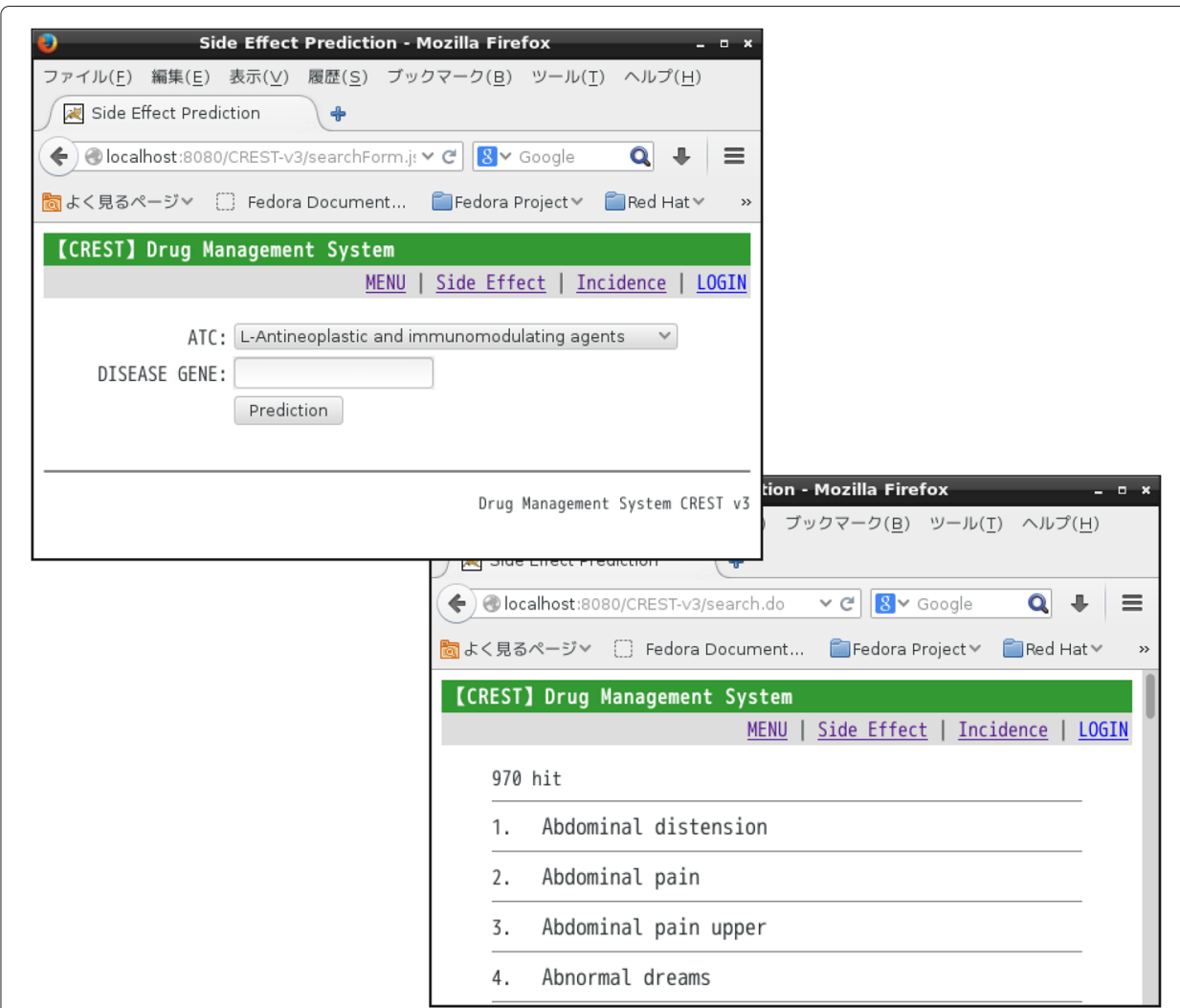

Fig. 9 Operation screen for side effect prediction. The left screen shows an input form for side effect prediction. The right screen displays the result which side effects are classified by 2 input items

cannot be predicted are as follows: 6 side effects are not nominated in this database; 7 side effects are not be classified although they are nominated in this database.

As for incidence prediction, there are 335 items to be predicted in side effect incidence. They are predicted according to each dataset shown in Table 2. Table 6 shows that $99.7 \%$ of 335 items in Dataset No. 1 gets the approximated intervals of incidence, and that $0.3 \%$ of them gets the higher intervals in the prediction. It will conclude that the gene information is useful for incidence prediction, and that the score as biological feature to extract regression characteristics of side effect contributes to predicting side effect incidence.

Thus, the proposed model can predict the approximation of side effect and its incidence. This is an important result of the prediction model. When clinical data of drugs are not prepared for drug screening, pinpoint data which shows drug properties are available for predicting side effect by the proposed model.

\section{Drug discovery by new uses for old drugs}

In the first approach of discovering drug candidates in new chemicals (drugs)-proteins (disease genes) interactions, the number of new uses for old drugs is evaluated in the proposed database application. The procedure of extracting new drug candidates from the database with SqlTool is as follows: first, to extract Gene ID and chemical ID pairs from Gene, Drug, and Disease tables; second, to extract Gene ID and chemical ID pairs in the drug candidate files searched by the extracted chemical ID; third, to extract 


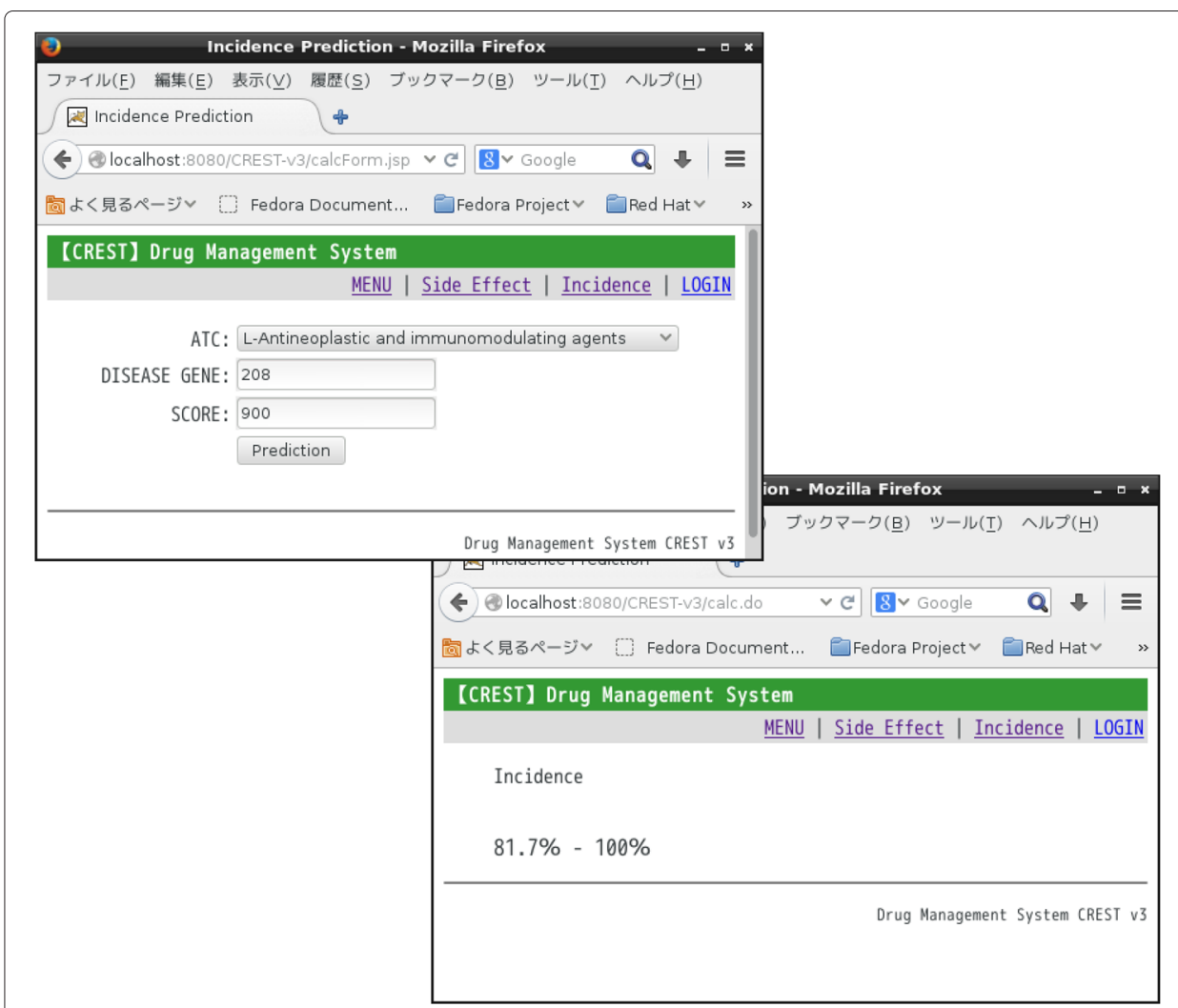

Fig. 10 Operation screen for incidence prediction. The left shows an input form for side effect incidence. The right screen displays the result which side effect incidence is calculated with 3 input items by LibSVM

new combinations by excluding Gene IDs nominated in the first result from Gene IDs nominated in the second result.

As a result, 32 new drug candidates of old drugs can be found. Among them, three drug candidates for three genes which has no drugs in the KEGG database and one drug candidate for one gene which has some drugs in the KEGG database are discovered as a therapeutic agent. The former three new drug candidates are "hydroxyurea" for NBN gene of primary immunodeficiency or congenital disorder of DNA repair systems, "probucol" for COG1 gene of inherited metabolic disease, and "pyridoxine" for SLC25A38 gene of

Table 6 Performance of incidence prediction

\begin{tabular}{lll}
\hline Dataset No. & Approximated interval & Higher interval \\
\hline 1 & $99.7 \%$ & $0.3 \%$ \\
2 & $97 \%$ & $3 \%$ \\
3 & $11 \%$ & $89 \%$ \\
4 & $98 \%$ & $2 \%$ \\
5 & $76 \%$ & $24 \%$ \\
6 & $71 \%$ & $29 \%$ \\
7 & $44 \%$ & $56 \%$ \\
\hline
\end{tabular}

"Dataset No". indicates 7 dataset patterns of pinpoint data shown in Table 2. "Approximated interval" indicates correct rate which a drug side effect incidence can be predicted within a correct clustering. "Higher interval" indicates that the predicted interval is higher than a correct interval of a drug side effect incidence 
hematologic disease. The latter one drug is "IMIQUIMOD" for TLR9 gene of immune system disease.

In the second approach of discovering drug candidates in the disease group related by disease genes and SNPs, the number of disease which can discover the new drug candidate is evaluated. The procedure of extracting diseases which can discover new drug candidate is as follows: first, to extract diseases which have the common SNPs or disease gene from two tables of "Gene" and "Jsnp"; second, when some diseases have the common SNP or disease gene, those diseases are related with each other and grouped with the grouping tool developed in Java.

As a result, 717 diseases in which 174 diseases have the drugs are classified into 187 groups. 93 groups have no drugs. 94 groups have some diseases which have some drugs. Then, 328 disease can discover the new drug candidates by the disease group.

\section{Conclusion}

This paper describes database application model and its service for drug discovery introducing our proposed software development process in MDA into our research process. The issue of veracity can be solved when pinpoint data are selected from drug properties in big data analytics with domain model. The datasets of pinpoint data are designed to predict side effect and its incidence even if some of pinpoint data are missing. The proposed prediction model of side effect and its incidence can attain almost $100 \%$ accuracy in its performance. In addition to our first research goal of side effect prediction in drug discovery, we can discover 2 new services for drug discovery by new uses for old drugs in logical system model with big data analytics. The veracity can be strengthened based on the number of disease gene directly interacted to chemicals (drugs) and the number of the disease gene and SNPs contributing to human disease. So far, it can be concluded that the reputation of data modeling with data analytics and implementation can strengthen the "veracity" of big data.

Our approach of software development process in MDA will be useful for developing a big data application and a new service by "veracity" and "value" of big data because MDA provides an approach for deriving value from models and architecture. Our proposal will contribute to the personalized drug discovery with drug screening of side effect prediction in the personalized medicine although our proposed system is comprehensive.

Competing interests

The author declare that she has no competing interests.

Authors' contributions

NE designed the study, developed the models, implemented the system, performed the analysis and evaluation and wrote the manuscript. And NE read and approved the final manuscript.

Acknowledgments

This work was supported by Japan Science and Technology Agency in a CREST research program entitled "Advanced

Application Technologies to Boost Big Data Utilization for Multiple-Field Scientific Discovery and Social Problem Solving".

Received: 28 May 2015 Accepted: 23 July 2015

Published online: 07 August 2015

References

1. Suresh J (2014) Bird's eye view on "big data management". In: Proceedings of 2014 Conference on IT in Business, Industry and Government (CSIBIG): 08-09 Mar 2014. IEEE: Madhya Pradesh Subsection, India. pp 1-5.

doi:10.1109/CSIBIG.2014.7056930 
2. Model Driven Architecture (MDA): The MDA Guide Rev 2.0, OMG Document Ormsc/2014-06-01 (2014). http://www. omg.org/cgi-bin/doc?ormsc/14-06-01

3. Repurposing Drug (2015). http://www.ncats.nih.gov/research/reengineering/rescue-repurpose/rescue-repurpose. html

4. Zhao XM, Iskar M, Zeller G, Kuhn M, van Noort V, Bork P (2011) Prediction of drug combinations by integrating molecular and pharmacological data. PLOS Comput Biol 7(12):e1002323. doi:10.1371/journal.pcbi.1002323

5. Rising Reports of Bad Reactions to Drugs Mar 282011 (2011). http://www.reuters.com/article/2011/03/28/us-risingreports-badreactions-drugs-idUSTRE72R65Z20110328

6. Tailor-made Medical Treatment Program (2015). http://www.biobankjp.org/english/leaflet/about.html

7. Yamanishi Y, Pauwels E, Kotera M (2012) Drug side-effect prediction based on the integration of chemical and biological spaces. J Chem Inf Model 52(12):3284-3292. doi:10.1021/ci2005548

8. Kuhn M, Al. Banchaabouchi M, Campillos M, Jensen L, Gross C, Gavin A, Bork P (2013) Systematic identification of proteins that elicit drug side effects. Mol Syst Biol. 9(663). doi:10.1038/msb.2013.10

9. Okada Y (2013) Genetics of rheumatoid arthritis contributes to biology and drug discovery. Nature 506(7488). doi:10.1038/nature12873

10. Rheumatoid Arthritis Research Shows the Potential of Large-scale Genetic Studies for Drug Discovery (2013). http:// www.riken.jp/en/pr/press/2013/20131226_1/

11. KEGG DISEASE (2014). http://www.genome.jp/kegg/disease/

12. Anatomical Therapeutic Chemical (ATC) Classification (2015). http://www.genome.jp/kegg-bin/get_htext?\& extend $=$ \&htext=br08303.keg

13. Hirakawa M, Tanaka T, Hashimoto Y, Kuroda M, Takagi1 T, Nakamura Y (2002) Jsnp: a database of common gene variations in the japanese population. Nucleic Acids Res 30(1):158-162

14. Haga H, Yamada R, Ohnishi Y, Nakamura Y, Tanaka T (2002) Gene-based snp discovery as part of the japanese millennium genome project : identification of 190,562 genetic variations in the human genome. J Hum Genet 47(11):605-610

15. JSNP DATABASE (2015). http://snp.ims.u-tokyo.ac.jp/

16. STITCH 4.0 (2014). http://stitch.embl.de/

17. Kuhn M, Campillos M, Letunic I, Jensen LJ, Bork P (2010) A side effect resource to capture phenotypic effects of drugs. Mol Syst Biol 6(343). doi:10.1038/msb.2009.98

18. SIDER 2 Side Effect Resource (2014). http://sideeffects.embl.de/

19. PubChem (2014). http://pubchem.ncbi.n/m.nih.gov/\#

20. Anatomical Therapeutic Chemical Classification System (2015). http://en.wikipedia.org/wiki/Anatomical_ Therapeutic_Chemical_Classification_System

21. LIBSVM A Library for Support Vector Machines (2014). https://www.csie.ntu.edu.tw/ cjlin/libsvm/

22. Book Management System (bcat) (2004). http://bcat.fujiwaralab.net/download.html

23. The Internet Drug Index, RxList Inc (2015). http://www.rxlist.com/script/main/hp.asp

\section{Submit your manuscript to a SpringerOpen ${ }^{\circ}$ journal and benefit from:}

- Convenient online submission

- Rigorous peer review

- Immediate publication on acceptance

- Open access: articles freely available online

- High visibility within the field

- Retaining the copyright to your article

Submit your next manuscript at $\gg$ springeropen.com 